\title{
The neurological content of general practice
}

\author{
THODOROS PAPAPETROPOULOS, EVAGELIA TSIBRE, VASSILIOS PELEKOUDAS
}

From the Department of Neurology, School of Health Sciences, University of Patras, Greece

SUMMARY Of examined patients in medical practice in this study $12.3 \%$ presented with neurological disease or health problems involving neurological questions or approach. In acute cases neurological problems $(15.5 \%)$, occurred more often than in chronic ones. This high neurological workload in general practice has to be taken seriously into consideration in undergraduate and postgraduate medical training.

There has been much discussion and debate on the role of neurology and the proper number and type of neurologists in medical practice. ${ }^{1-8}$ The need to learn more about the neurological content of different aspects of internal medical practice has often been stressed.

We present a study which tries to explore prospectively the ratio of neurological diseases or health problems posing neurologic questions, in chronic and acute medical cases.

\section{Material and methods}

We used three different sources of patient: (a) National rural health offices or centres (NRHO, NRHC) in Achaia district of Peloponese and (b) urban health centres of Institute of Social Security (UHCISS) in the city of Patras, mainly for chronic medical cases, and (c) the emergency out patient medical department (EOPMD) of a district general hospital (Agios Andreas, Patras) for acute cases.

We asked physicians in NRHO, NRHC and UHCISS to complete a protocol with the age, sex, and health problem(s), for every consecutively examined patient. In NRHO-NRHC physicians work after graduation without postgraduate training in specialties; in UHCISS the staff are generalistsinternists, while in Agios Andreas Hospital they are internists included neurologists. The study period was 8 weeks for NRHO-NRHC (March-April 1987) 8 weeks for UHCISS

Address for reprint requests: Dr T L Papapetropoulos, Department of Neurology, Agios Andreas Hospital, Petra 26110, PO Box 1045, Greece.

Received 25 May 1988.

Accepted 8 November 1988

Table Neurological content of general practice
(May-June 1987) and 3 months for EOPMD (February, June, October 1986).

We received completed protocol forms from 9 NRHO, (between 41), 1 NRHC (between 3) with 4107 examined persons, and from 12 UHCISS doctors, (between 45) with 7859 examined.

One of us (T E trainee neurologist) working in Agios Andreas Hospital EOPMD, completed the same protocol from the official emergency out patient medical department records for 6055 consecutively examined patients during the study period.

The first source of cases included children and adults while the second and third only adults. As in other surveys terminology varied between reporting physicians; under the column "health problem" there were complaints, symptoms, syndromes or diseases. Some symptoms such as vertigo low back pain, functional disturbances headache, quite often noticed by the physicians, were not exclusively neurological, but have been considered as "neurological" because they need mainly neurological questions and examination.

\section{Results}

Among 18021 examined persons $12.3 \%$ presented with neurological disease or health problems which needed neurological enquiry or approach ("neurological symptoms). The table shows the percentage of neurological disease or symptoms in rural health centres and offices in urban health centres and in emergency medical out patients departments. In the first two sources of patients, with mainly chronic cases, the percentage was about the same but quite lower in relation to the third source with the acute cases. Headache, vertigo, functional disturbances, epilepsy,

\begin{tabular}{lccc}
\hline Source of patients & No examined & $\begin{array}{l}\text { Patient with "neurological" symptom } \\
\text { or disease }\end{array}$ & $\begin{array}{l}\text { Percentage of patient with neurological } \\
\text { symptom or disease }\end{array}$ \\
\hline $\begin{array}{lcc}\text { (a) Rural health offices centres } \\
\text { (b) Urban health centres }\end{array}$ & 4107 & 431 & $10 \cdot 49 \%$ \\
(c) Emergency out patient medical & 7859 & 860 & $10.94 \%$ \\
$\begin{array}{l}\text { department } \\
\text { Total }\end{array}$ & 6055 & 933 & $15.50 \%$ \\
\hline
\end{tabular}


low back pain and sciatica were the most often recorded health problems.

\section{Discussion}

Of examined patients in rural and urban primary medical practice in this study $10.7 \%$ presented with neurological disease or symptoms which needed a neurological enquiry and approach. In a similar study in Nova Scotia ${ }^{9}$ approximately $10 \%$ of cases had presented with symptoms suggesting neurological problems. In Virginia in $1976^{10} 7 \%$ of examined patients had neurological problems while in a more recent study in $1986^{11}$ neurological questions were addressed in $9 \%$ of patient visits. In acute adult patients examined in emergency out patient medical departments of this study the percentage of neurological disease or symptoms was higher (15.5\%).

Undergraduate medical curriculum planners and determinators those responsible for training of generalist internists must take seriously into account the above remarkable neurological workload of general practice. A short period of training in regional neurological departments (for example 6 months) for internists and generalists might increase their ability to cope with neurological symptoms and problems in their practice. Headache, back pain, vertigo, function disturbances, cerebrovascular accidents, which were the more common neurologic problems in our study should be the main topics in training programmes for neurology.

\section{References}

1 Hopkins A. Different types of neurologists. Br Med J 1984;288:1733-6.

2 Menken M. The scope of Neurological practice: evidence from a practice study. Arch Neurol 1985;42:386-7.

3 Kurtzke JF. The current neurological burden of illness and injury in the United States. Neurology 1982;32: 1207-14.

4 Morrow JI, Patterson VH. The neurological practice of a district general hospital. J Neurol Neurosurg Psychiatry 1987;50:1397-401.

5 Dyken ML. The continuing undersupply of neurologists in the 1980's; impressions based on data from three studies. Neurology 1982;32:651-6.

6 Garrison LP, Bowman MA, Perrin EB. Estimating physician requirements for neurology: a needs-based approach. Neurology 1984;34:1218-27.

7 Menken M. The coming oversupply of neurologists in the 1980's. JAMA 1981;245:2401-3.

8 O'Doherty DS. National need for neurologists: commentary on the GMENAC report. Neurology 1984;34: 1228-30.

9 Murray TJ. Concepts in undergraduate neurological teaching. Clin Neurol Neurosurg 1976;79:275-84.

10 Marsland DW, Wood M, Mayo F. The content of family practice. J Fam Pract 1976;3:23-74.

11 Miller JR. The neurologic content of family practice. Implications for neurologists. Arch Neurol 1986;43: 286-8. 